\title{
Prevalence of Bifid Median Nerve in Patients with Moderate to Severe Carpal Tunnel Syndrome
}

\author{
Authors

\section{Dr Sreejith Kalathummarath ${ }^{1}$, Dr Muhlisa. V $^{2}$, Dr Shehadad Kammili ${ }^{3}$,} \\ Dr Sreedevi Menon. $\mathbf{P}^{4}$ \\ ${ }^{1}$ Associate Professor, ${ }^{2}$ Senior Resident, ${ }^{3}$ Assistant Professor, ${ }^{4}$ Professor and Head
} Dept of Physical Medicine and Rehabilitation, Government Medical College, Kozhikode, Kerala, India Corresponding Author

Dr Sreejith Kalathummarath

Associate Professor, Department of Physical Medicine and Rehabilitation,

Government Medical College, Kozhikode 673008

Email: sreejithpmr@gmail.com

\begin{abstract}
Introduction: Carpal tunnel syndrome is the entrapment neuropathy of median nerve at wrist. Bifid median nerve is a variation in the normal anatomy and can be associated with carpal tunnel syndrome. We aim to study the prevalence of bifid median nerve in patients with moderate to severe carpal tunnel syndrome.

Methods: An observational study was done in 74 patients with moderate to severe carpal tunnel syndrome for anatomic variations in the median nerve. The ultrasound evaluation of the wrist was performed in all patients. The datas were statistically analysed.

Results: The bifid median nerve was present in $12.2 \%$ and persistent median artery in $4.1 \%$ of the patients.

Conclusion: The bifid median is relatively common in patients with CTS. The ultrasound evaluation of the carpal tunnel is recommended in all patients with CTS.

Keywords: Carpal tunnel syndrome, bifid median nerve, persistent median artery.
\end{abstract}

\section{Background}

Carpal tunnel syndrome is the most frequent entrapment neuropathy in upper extremity ${ }^{1}$. It occurs due to the entrapment of median nerve in the carpal tunnel. Bifid median nerve is a variation in the anatomy of median nerve. ${ }^{2,}{ }^{3}$ It is the high division of median nerve proximal to the carpal tunnel. The variation in the anatomy of median nerve has been described by Lanz and classified into four groups. The bifid median nerve is classified under the group III of the Lanz classification. Bifid median nerves may be associated with a persistent median artery or an accessory muscle. ${ }^{4,5}$ The persistent median artery may dilate or thrombose to cause CTS. CTS is most commonly associated with group III of the Lanz classification. The bifid median has an increased risk for causing CTS as they occupy larger cross sectional area of carpal tunnel. ${ }^{3}$

The CTS is diagnosed by a clinical history, physical examination and confirmed with electrodiagnostic studies and ultrasonography. 
Ultrasonography is an emerging investigation for the diagnosis of CTS and also for planning the management ${ }^{6}$. The criteria for diagnosis of CTS based on USG is by assessing the cross sectional area of median nerve at the level of pisiform bone. The CSA of $10 \mathrm{~mm}^{2}$ or more in non bifid median nerve and $11 \mathrm{~mm}^{2}$ or more in case of bifid median nerve. $^{3,6,7}$ The CTS is managed conservatively initially followed by surgical methods if no relief. The release of transverse carpal ligament is the definitive surgical approach for moderate to severe CTS. ${ }^{8}$ USG helps us to delineate the anatomy of median nerve pre operatively. It helps us to rule out the anatomic variants, the proximity of blood vessels and also to plan the surgical incision site.

The prevalence of bifid median nerve is described inadequately. The prevalence ranges from $2 \%$ to $26 \% .^{3,9-12}$ In this study, we aim to find out the prevalence of bifid median nerve in moderate to severe CTS.

\section{Materials and Method}

The study was conducted in diagnosed cases of moderate to severe CTS posted for surgical release attending the department of Physical Medicine \& Rehabilitation, Government Medical College, Kozhikode. A cross sectional study was carried out in 74 patients of both gender aged between 18 to 65 years. Persons with history of wrist surgery, wrist injuries, cervical radiculopathy, polyneuropathies, inflammatory arthritis and pregnancy were excluded. Informed written consent was obtained from all the participants prior to the study. The study was approved by the institutional ethics committee.

The patient characteristics like age, sex, occupation, side of involvement, handedness, BMI were recorded. A detailed clinical history and followed by examination was done. The ultrasound examination of the wrist was performed by a physiatrist who has experience in musculoskeletal ultrasongraphy using the esaote MyLab 40 ultrasound machine. The contents of the carpal tunnel were visualized using high frequency linear probe of 10-18 MHz. The patients were seated in a chair keeping their hands on an examination couch with elbow extended, wrist in $20^{\circ}$ extension and fingers semiflexed. USG evaluation of the median nerve by transverse view was used to assess the cross sectional area (CSA). The probe is kept at the distal wrist crease at the level of pisiform bone and it is angulated in such a way that flexor tendons are hyperechoic.

The median nerve is located superficial to the echogenic flexor tendons by its characteristic honey comb or stippled appearance. The course of the median nerve in the tunnel was evaluated. The bifid median nerve was visualized as the branching of median nerve proximal to the distal radio ulnar joint. It appeared as two echogenic rims with honeycomb or stippled appearance. The cross sectional area of the median nerve was assessed by direct tracing excluding the echogenic rim. ${ }^{13}$ In case of bifid median nerve both trunks were traced separately and the values added up. ${ }^{3}$ The persistent median artery was found between the two trunks of bifid median nerve. Doppler sonography was done to confirm the presence of persistent median artery.

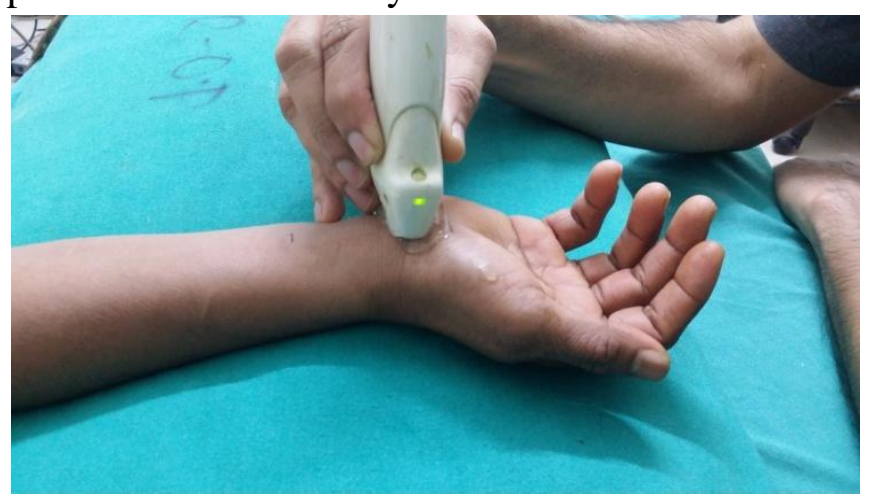

Figure: 1 Ultrasound examination of wrist

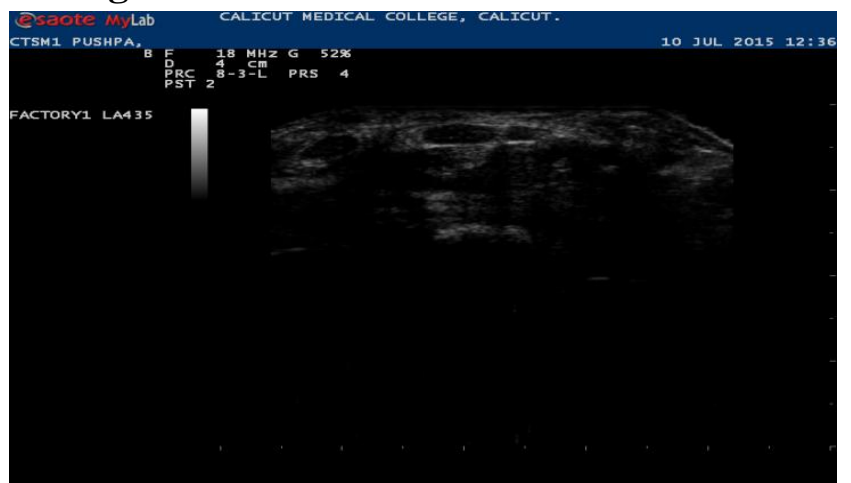

Figure: 2 Ultrasonogram of wrist showing Bifid median nerve 


\section{JMSCR Vol||05||Issue||10||Page 29638-29641||October}

\section{Statistical Analysis}

The statistical analysis was done by coding the data in excel sheet and analysed using SPSS 18.0. Qualitative variables are presented as frequency and percentages and comparison done using Chi square test. Quantitative variables are presented as mean and standard deviation.

\section{Results}

In our study we observed bifid median nerve in $12.2 \%$ of the patients and persistent median artery in $4.1 \%$.

\begin{tabular}{|l|c|c|c|c|}
\hline & Minimum & Maximum & Mean & SD \\
\hline AGE & 25 & 64 & 45.54 & 9.164 \\
\hline BMI & 20.17 & 34.6 & 25.7 & 3.101 \\
\hline CSA $\left(\mathbf{m m}^{2}\right)$ & 13 & 43 & 19.23 & 5.656 \\
\hline
\end{tabular}

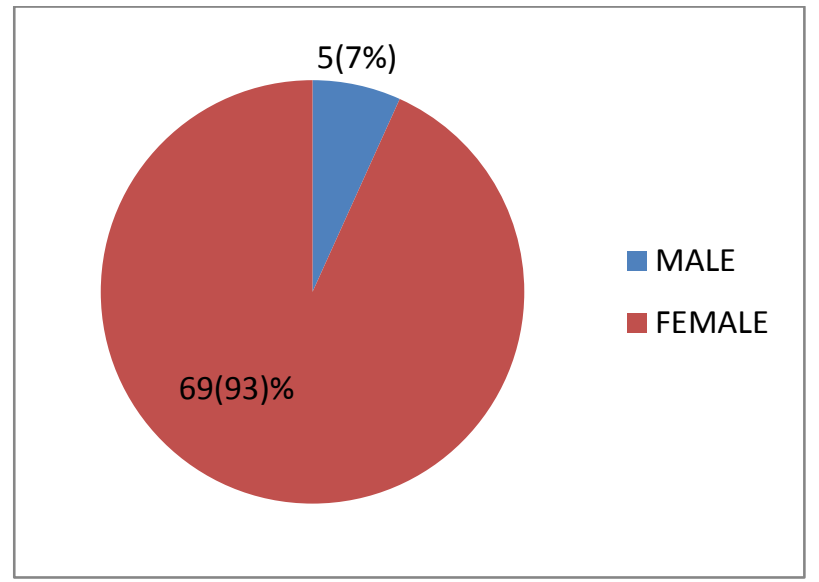

Figure: 3 Sex distributions

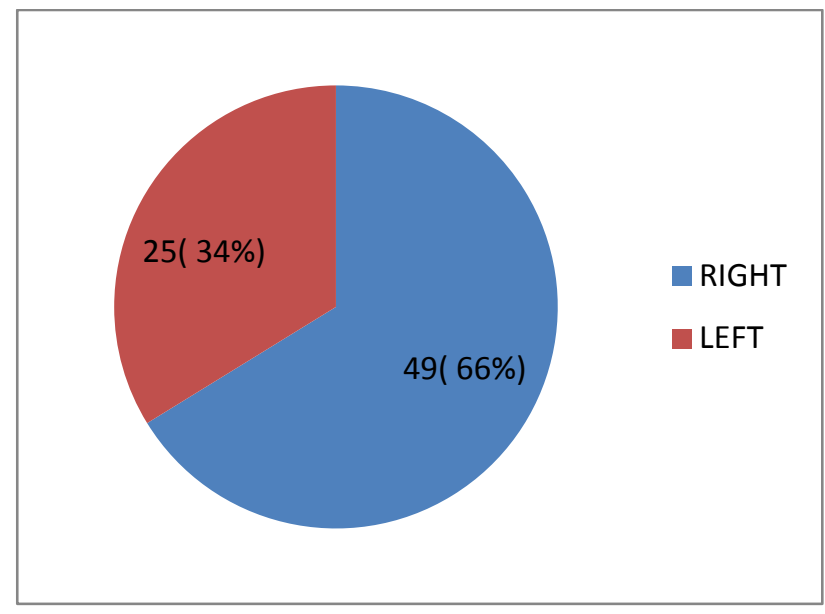

Figure: 4 Side of involvement

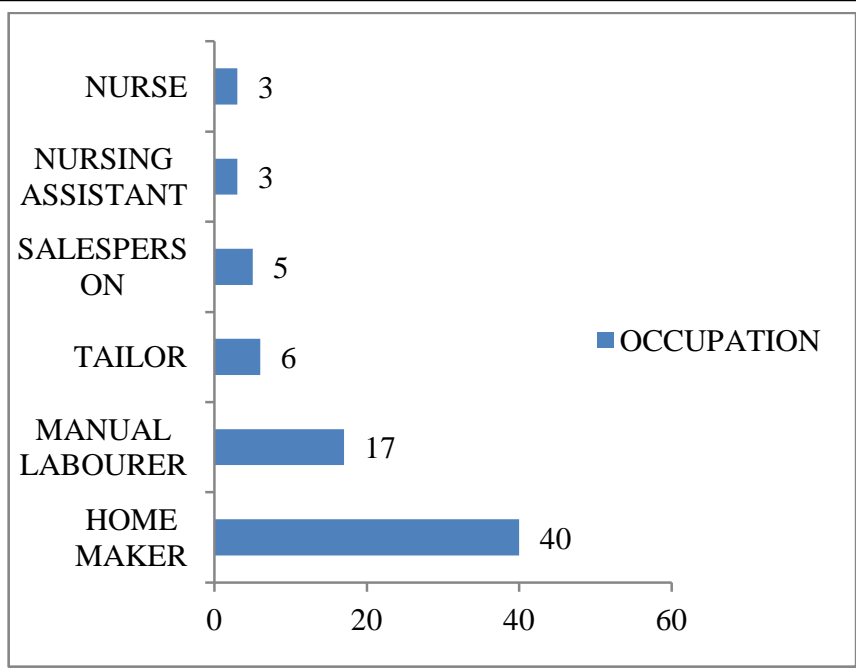

Figure: 5 Frequency of occupation

\section{Summary and Conclusion}

The study was done to find out the prevalence of bifid median nerve in moderate to severe CTS. We performed the ultrasound examination of the wrists of 74 subjects with CTS. Bifid median nerve was found in 9 patients with a prevalence of $12.2 \%$. Four patients had bilateral bifid median nerve. Persistent median artery was found in 3 patients with a prevalence of $4.1 \%$. The prevalence of bifid median nerve reported in previous studies ranged from $2 \%$ to $26 \%^{3,9-12}$ which is consistent with our findings.

Bayrak et al had reported that bifid median nerve occurs more frequently in persons with CTS. The bifid median nerve has higher cross sectional area compared to non bifid median nerve; hence it may facilitate the entrapment of median nerve in the carpal tunnel. Many studies have reported the importance of ultrasonography of the median nerve for anatomic variations and part of preoperative work up to avoid injury to median nerve, the blood vessels and inadequate decompression during surgery.

The major limitation of our study was the small sample size and absence of control group for comparison.

We conclude that bifid median nerve is relatively common in patients with CTS. The ultrasound evaluation of the carpal tunnel is recommended in all patients with CTS.

Source of support: Nil 


\section{References}

1. Alfonso C, Jann S, Massa R, Torreggiani A. Diagnosis, treatment and follow-up of the carpal tunnel syndrome: a review. Neurolog Sci 2010; 31(3): 243-52

2. Lanz U. Anatomical variations of the median nerve in the carpal tunnel. J Hand Surg [Am] 1977; 2:44-53.

3. Bayrak IK, Bayrak AO, Kale M, Turker H, Diren B. Bifid median nerve in patients with carpal tunnel syndrome. J Ultrasound Med. 2008; 27:1129-1136. [PubMed: 18645070]

4. Fernandez-Garcia S, Pi-Folguera J, Estallo-Marino F: Bifid median nerve compression due to a musculotendinous anomaly of FDS to the middle finger. J Hand Surg [Br] 19:616, 1994

5. Schultz RJ, Endler PM, Deneys Huddleston H:Anomalous median nerve and an anomalous muscle belly of the first lumbrical associated with carpal-tunnel syndrome. J Bone Joint Surg [Am] 55:1744, 1973

6. Naranjo A, Ojeda S, Mendoza D, Francisco F, Quevedo Jc, Erausquin C: What is the diagnostic value of ultrasonography compared to physical evaluation in patients with idiopathic carpal tunnel syndrome? Clin Exp Rheumatol 2007; 25: 853-9

7. Presazzi A, Bortolotto C, Zacchino M, Madonia L, Draghi F. Carpal tunnel: Normal anatomy, anatomical variants and ultrasound technique. Journal of ultrasound. 2011 Mar 31;14(1):40-6

8. Dawson DM. Entrapment neuropathies of the upper extremities. New England Journal of Medicine. 1993 Dec 30;329(27):2013-8.

9. Pierre-Jerome C, Smitson RD Jr, Shah RK, Moncayo V, Abdelnoor M, Terk MR. MRI of the median nerve and median artery in the carpal tunnel: prevalence of their anatomical variations and clinical significance. Surg Radiol Anat. 2010; 32:315-322. [PubMed: 20033168]

10. Granata G, Caliandro P, Pazzaglia C, Minciotti I, Russo G, Martinoli C, et al. Prevalence of bifid median nerve at wrist assessed through ultrasound. Neurol Sci. 2011; 32:615-618. [PubMed: 21533564]

11. Lannicelli E, Chianta GA, Salvini V, Almberger M, Monacelli G, Passariello R. Evaluation of bifid median nerve with sonography and MR imaging. J Ultrasound Med. 2000; 19:481-485. [PubMed: 10898302]

12. Padua L, Liotta G, Di Pasquale A, Granata G, Pazzaglia C, Caliandro $P$, et al. Contribution of ultrasound in the assessment of nerve diseases. Eur $\mathbf{J}$ Neurol. 2012; 19:47-54. [PubMed: 21554493]

13. Chen SF, Huang CR, Tsai NW, Chang CC, $\mathrm{Lu} \mathrm{CH}$, Chuang $\mathrm{YC}$, et al. Ultrasonographic assessment of carpal tunnel syndrome of mild and moderate severity in diabetic patients by using an 8point measurement of median nerve crosssectional areas. BMC Med Imaging. 2012;12:15.
Abbreviations
CTS- Carpal Tunnel Syndrome
PMA- Persistent median artery
USG- Ultrasonography
BMI- Body mass index
CSA- Cross sectional area 